\title{
Features of Barrier Capacitance of Micropixel Avalanche Photodiodes at Different Frequencies
}

\author{
E.A. Jafarova ${ }^{1, *}$, Z.Y. Sadygov ${ }^{1,3}$, F.I. Ahmadov ${ }^{2}$, A.Z. $\operatorname{Sadygov}^{2}$, A.A. Dovlatov ${ }^{1}$, \\ L.A. Aliyeva ${ }^{1}$, E.S. Taptygov ${ }^{1}$ \\ ${ }^{1}$ Institute of Physics, Azerbaijan National Academy of Sciences, Azerbaijan \\ ${ }^{2}$ Institute of Radiation Problems, Azerbaijan National Academy of Sciences, Azerbaijan \\ ${ }^{3}$ Joint Institute for Nuclear Research, Russia
}

Copyright $(2016$ by authors, all rights reserved. Authors agree that this article remains permanently open access under the terms of the Creative Commons Attribution License 4.0 International License

\begin{abstract}
There has been investigated the capacitance of silicon micro pixel avalanche photodiodes (MAPD 3N) with deeply buried pixels under the effect of weak $A C$ signal of different frequency (from $10 \mathrm{kHz}$ to $1 \mathrm{MHz}$ ). A decreasing of the barrier capacitance with an increasing of AC signal frequency has been observed when small DC bias voltage $(0-3 \mathrm{~V})$ is applied to the structure. With the rise of voltage the observed dependence weakens and further saturates. It is established that capacity behavior like this within small voltage is referred to the peculiarity of MAPD structure under the investigation: presence of matrix of $\mathrm{n}^{+}$-regions between two epitaxial layers of p-type conductivity results in the appearance of some effective resistance between these layers connected in series with the measured capacity depending on AC signal frequency. The calculated values of ionized acceptor concentration from the slope of the dependence $\mathrm{C}^{-2}\left(\mathrm{U}_{\text {bias }}\right)$ in epitaxial layer are: $\mathrm{N}_{\mathrm{A} 1}=(2.4 \pm 0.3) \cdot 10^{20} \mathrm{~m}^{-3}$ for $\mathrm{U}_{\text {bias }}$ up to $3 \mathrm{~V}$, $\mathrm{N}_{\mathrm{A} 2}=(1.08 \pm 0.05) \cdot 10^{21} \mathrm{~m}^{-3}$ for $\mathrm{U}_{\text {bias }}$ from 3 to $10 \mathrm{~V}$ and $\mathrm{N}_{\mathrm{A} 3}=(2.13 \pm 0.27) \cdot 10^{21} \mathrm{~m}^{-3}$ for $\mathrm{U}_{\text {bias }}$ from 10 to $20 \mathrm{~V}$ respectively.
\end{abstract}

Keywords Avalanche Photodiode, Capacitance of $p-n$ Junction, Space Charge Layer, Concentration of Ionized Impurities

\section{Introduction}

Recently developed micropixel avalanche photodiodes (MAPD) [1-4], with a high gain $\left(\sim 10^{5}\right)$ and high efficiency of a single light quanta registering (40\%), are widely used in modern devices. MAPD are widely utilized in equipment for scientific researches, especially in nuclear medical equipment, physical equipment, and radiation monitoring.

The manufacturing technology and the physical processes of the micropixel avalanche photodiodes with deeply buried pixels has been described earlier [4-8]. MAPD structure comprises silicon n-type substrate and two epitaxial layers of p-type conductivity have been grown on its surface. A matrix of $\mathrm{n}^{+}$- areas of 2-5 microns has been arranged between two p-type epitaxial layers. The matrix is made by ion implantation of the first $\mathrm{p}$-type epitaxial layers. The $\mathrm{n}^{+}-$areas are spaced by necessary intervals (3-5 micron) to prevent the charge connection among them. A segmentation of $\mathrm{p}-\mathrm{n}^{+}$ junctions to disconnected areas allows increasing of a reverse biasing voltage above breakdown i.e. operate in the overvoltage mode. The self-quenching Geiger discharge has been evolved when a light quantum hits the active region of any pixel. In avalanche diodes with deeply buried pixels quenching of discharge occurs due to the fall of voltage in $\mathrm{p}-\mathrm{n}$ junction below breakdown, by accumulating part of multiplied electrons in potential well (each micropixel contains potential well because of $p-n^{+}-p$ structure). The recovery of previous electric field in multiplication micro channels is taken place due to running off the accumulated charge to the substrate through a forward-biased $p-n$ junction between the first epitaxial layer and the $\mathrm{n}^{+-}$- region.

Presence of high-doped $\mathrm{n}^{+}$- regions between two p-type epitaxial layers in MAPD with deeply buried pixels leads to a particular potential distribution in the depletion region [9]. Earlier capacitive properties of avalanche photodiodes with deeply buried pixels have been considered in [10]. It was shown that in structures under the investigation of three series connected $p-n$ junctions, the total capacitance measured as a function of bias voltage (positive potential is applied to $\mathrm{n}-\mathrm{Si}$ substrate with $\rho=7 \mathrm{Ohm} \cdot \mathrm{cm}$ ) is $\mathrm{C}_{\text {tot }}=1 / 2 \mathrm{C}_{1}$, where $\mathrm{C}_{1}$ is the capacitance of first $\mathrm{n}^{+}-\mathrm{p}$ junction.

Measurements have been carried out at a frequency $\mathrm{f}=10 \mathrm{MHz}$. It is known that the frequency of AC signal superposed on a large DC bias has a significant influence on the physical processes taking place in the $p-n$ junction, particular for recovery of device parameters.

Thus it is of great interest to investigate the dependence of measured barrier capacitance MAPD structures on the frequency of the AC signal along with its influence on the measured value of the width of the space-charge layer and the calculated concentration of ionized acceptors. 


\section{Materials and Methods}

\subsection{Techniques of Measurement}

The setup for measuring diode's capacitance is presented in Figure 1. AC signal with constant amplitude of $40 \mathrm{mV}$ is applied to a sample by a pulse generator Textronix AFG 3102. The frequency of the AC pulse varies from $10 \mathrm{kHz}$ to $1 \mathrm{MHz}$. Passed through the MAPD signal is taken by oscilloscope DP07254 from resistor $R_{3}$. The resistance of $R_{3}$ was selected such that the amplitude of signal would not be higher than $2.5 \mathrm{mV}$. Due to this the resistance of $\mathrm{R}_{3}$ should be selected as $1 \mathrm{k} \Omega, 257 \Omega, 60 \Omega, 30 \Omega$ and $11.3 \Omega$ for the frequencies $10 \mathrm{kHz}, 30 \mathrm{kHz}, 100 \mathrm{kHz}, 600 \mathrm{kHz}$ and $1 \mathrm{MHz}$ respectively (The properties of the differentiating circuit were taken into account). In this case the accuracy of measurement of capacity is within $7 \%$.

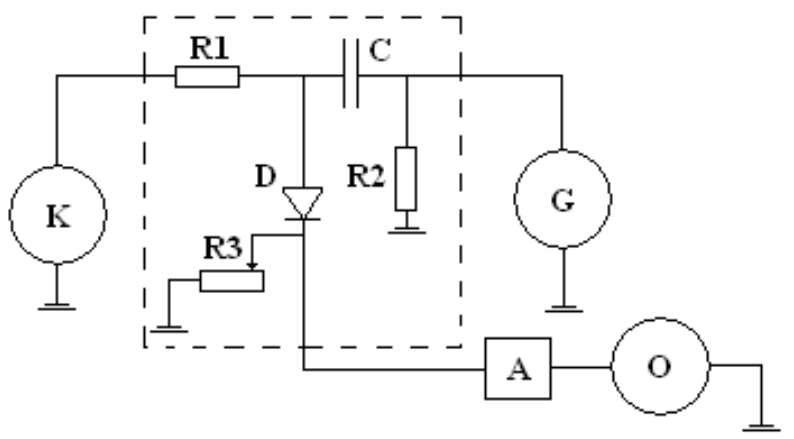

Figure 1. Capacitance measurement circuit diagram for micropixel avalanche photodiodes: K -power supply; G - generator; A - amplifier; O oscilloscope.

Pre-calibration of the setup was carried out by using standard high-frequency capacitors 220 and $440 \mathrm{pF}$, included in the circuit instead of the diode and the voltage drop $\mathrm{U}_{\text {ref }}$ was determined. The capacity of tested MAPD decreases with bias voltage, so for accurate measurement of photodiode capacitance $C_{D}$ in the range $U_{\text {bias }}=(0-2) \mathrm{V}$ gauge capacitance $\mathrm{C}_{\text {ref }}=440 \mathrm{pF}$, and for $\mathrm{U}_{\text {bias }}=(2-20) \mathrm{V}-\mathrm{C}_{\mathrm{ref}}=$ $220 \mathrm{pF}$ have been selected.

MAPD-3N of working voltage $\mathrm{U}=90.2 \mathrm{~V}$ and photodetection efficiency $\mathrm{PDE}=30 \%$ is chosen as a test sample. DC voltage applied by power supply Keithley $64871 \mathrm{~F}$. The capacitance of MAPD determined from the relationship:

$$
\mathrm{C}_{\mathrm{D}}=\left(\mathrm{U}_{\mathrm{D}} / \mathrm{U}_{\text {ref }}\right) \cdot \mathrm{C}_{\mathrm{ref}}
$$

where $U_{D}$ - voltage drop across the load $R_{3}$ when both the $D C$ voltage and AC pulse are applied.

\subsection{Results and Discussion}

As it is mentioned in [9], voltage dependence of the barrier capacitance of $p-n$ junction prepared by epitaxial method is better described by the expression for the case of a sharp distribution of the concentration of impurities. Thus the concentration of acceptor impurities is:

$$
N_{A}=\frac{2 d\left(U_{\text {bias }}\right)}{S^{2} q \varepsilon \varepsilon_{o} d\left(1 / C_{D}^{2}\right)}
$$

where S-area of p-n junction, $\varepsilon$ - semiconductor permittivity, $\varepsilon_{\mathrm{o}}$ is the dielectric constant of vacuum $\left(\varepsilon_{\mathrm{o}}=8.85 \cdot 10^{-12} \mathrm{~F} / \mathrm{m}\right)$, q- electron charge, $\mathrm{U}_{\text {bias }}$-applied voltage, $\mathrm{C}_{\mathrm{D}}$-capacitance of the diode.

Measurement results of voltage dependence of barrier capacitance of the MAPD $3 \mathrm{~N}$ with applied AC signal at various frequencies are presented in Figure 2.

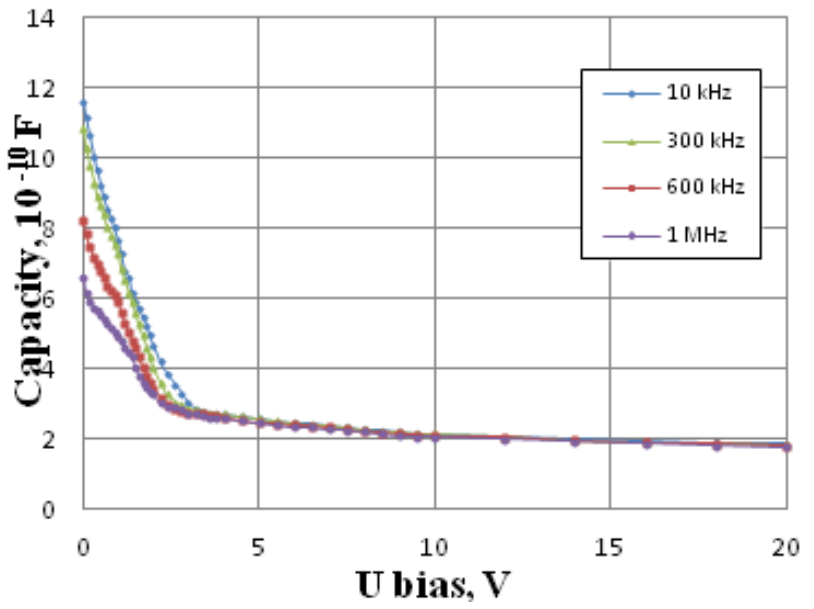

Figure 2. Dependence of MAPD $3 \mathrm{~N}$ photodiode capacitance on the applied voltage at different frequencies.

At small values of $U_{\text {bias }}=0-3 B$ the observed decrease of capacitance is most strongly expressed at frequency $\mathrm{f}=10$ $\mathrm{kHz}$, and weaker at the largest frequency $\mathrm{f}=1 \mathrm{MHz}$. At high $\mathrm{U}_{\text {bias }}$ relation of capacitance $\mathrm{C}$ vs $\mathrm{U}_{\text {bias }}$ saturates and is almost independent of frequency.

The frequency dependence of capacitance at low voltage $(0-3 \mathrm{~V})$ related to structural features of the investigated MAPD sample. The presence of array of $\mathrm{n}^{+}$-regions between two epitaxial layers of p-type conductivity leads to an existence of an effective resistance between epitaxial layers. As the depletion begins from the boundary of substrate and first epitaxial layer, the above mentioned effective resistance is connected in series with the measured capacitance. Of course, the influence of this resistance on the measured capacitance value depends on the frequency of AC signal: the higher the frequency, the stronger is the influence. This is confirmed by the results shown in Fig. 3. 


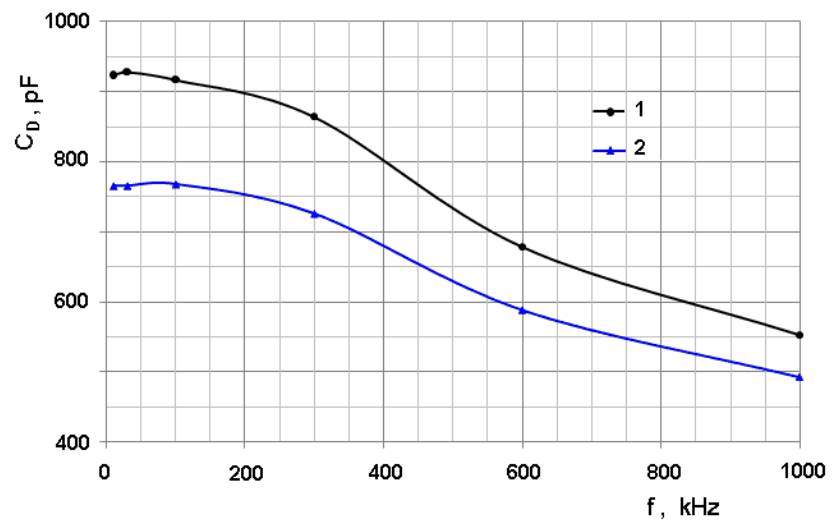

Figure 3. Frequency dependence of measured capacitance magnitude at two fixed applied voltage. $1-\mathrm{U}_{\text {bias }}=0.5 \mathrm{~V} ; 2-\mathrm{U}_{\text {bias }}=1 \mathrm{~V}$.

As it seen from Fig. 3 within the frequency range 10-100 $\mathrm{kHz}$ the capacity of the device remains constant. It indicates the fact that the reactance of photodiode is significantly more than the magnitude of series resistance and therefore the measured capacitance is equal to the barrier capacitance of device.

The width of space-charge layer, determined from capacitance measurement in range $\mathrm{U}_{\text {bias }}=0-3 \mathrm{~B}$ depends on frequency and increases sharply from 0.8 to $2.9 \mu$ for frequency $=10 \mathrm{kHz}$ and from 1.4 to $3.45 \mu$ for frequency $=$ $1 \mathrm{MHz}$.

At relatively higher voltages $U_{\text {bias }}(3-20 \mathrm{~V})$, it reaches the value 5 microns and almost does not depend on frequency (Fig. 4, Tab. 1).

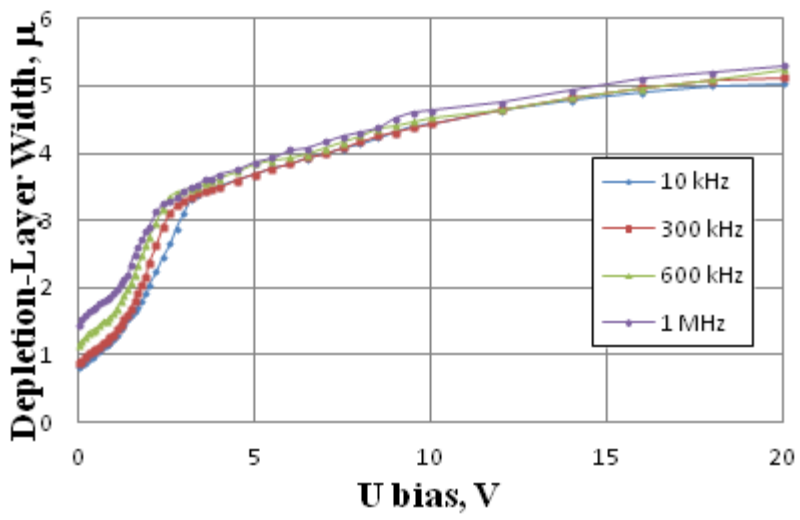

Figure 4. Dependence of the width of the space charge layer MAPD $3 \mathrm{~N}$ vs. $\mathrm{U}_{\text {bias }}$ at different frequencies.
Depending on $\mathrm{C}^{-2}\left(\mathrm{U}_{\text {bias }}\right)$ there have been observed three slopes from wich by formula (1) the concentration of ionized acceptors is determined (Fig. 5): $\mathrm{N}_{\mathrm{Al}}=(2.4 \pm 0.3) \cdot 10^{20} \mathrm{~m}^{-3}$ at $\mathrm{U}_{\text {bias }} 0-3 \mathrm{~V}, \mathrm{~N}_{\mathrm{A} 2}=(1.08 \pm 0.05) \cdot 10^{21} \mathrm{~m}^{-3}$ at $\mathrm{U}_{\text {bias }}=3-10 \mathrm{~V}$ and $\mathrm{N}_{\mathrm{A} 3}=(2.13 \pm 0.27) \cdot 10^{21} \mathrm{~m}^{-3}$ at $\mathrm{U}_{\text {bias }}=10-20 \mathrm{~V}$. As it is seen from Fig. 5 the impurity concentration defined from the slope $\mathrm{C}^{-2}\left(\mathrm{U}_{\text {bias }}\right)$ does not almost depends on AC signal frequency (Table 1). As the $\mathrm{C}^{-2}\left(\mathrm{U}_{\text {bias }}\right)$ dependence has three slopes the capacitance voltage of the cutoff $U_{\text {cut }}$ defined from these measurements takes different values not corresponding to the real ones.

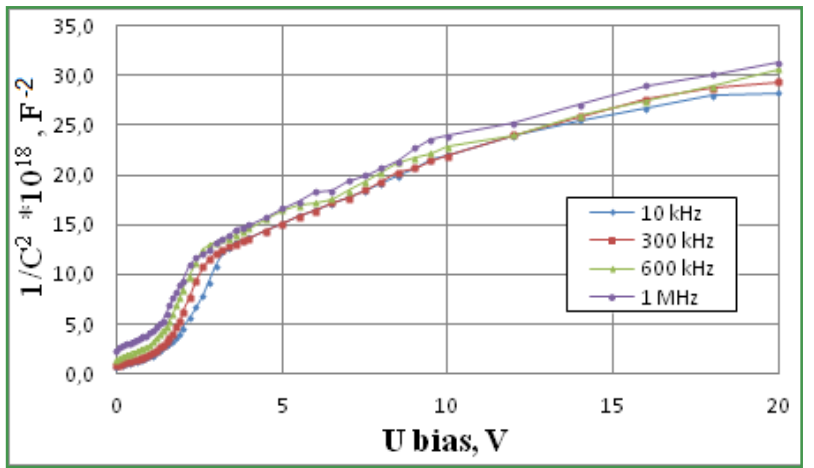

Figure 5. Dependence $\mathrm{C}^{-2}\left(\mathrm{U}_{\text {cut }}\right)$ for MAPD $3 \mathrm{~N}$ at different frequencies AC signal ( $\mathrm{f}=10 \mathrm{kHz}-1 \mathrm{MHz})$.

In summary, the equivalent circuit of the structure under investigation is the complex (three series p-n junction, where two outer ones are reverse-bias but the middle one is forward-bias) therefore it is not possible to explain clearly the contributions of each of the $3 \mathrm{p}$-n junctions in total measured capacitance. Furthermore, determination of the cutoff voltage $U_{\text {cut }}$ obtained from extrapolation of slope line of $\mathrm{C}^{-2}\left(\mathrm{U}_{\text {cut }}\right)$ to the point of intersection with the voltage axis is not correct enough.

The upper and lower epitaxial layers have no ohmic connection between themselves at the $\mathrm{n}^{+}$- matrix area.

Ohmic connection between them is carried out only through lateral areas of the device (beyond the $\mathrm{n}^{+}$- matrix area). It leads to the appearance of big surface conductivity of the lower epitaxial layer that has an effect on the inaccuracy in the measurements of dependence $\mathrm{C}$ on $\mathrm{U}_{\text {bias }}$ of the device.

Table 1. Calculated values of the space charge layer width and concentration of ionized acceptors depending on AC signal frequency.

\begin{tabular}{|c|c|c|c|c|c|}
\hline \multirow{2}{*}{$\mathrm{f}, \mathrm{kHz}$} & $\mathrm{U}_{\text {bias }}=0-3 \mathrm{~V}$ & $\mathrm{U}_{\text {bias }}=3-20 \mathrm{~V}$ & $\mathrm{U}_{\text {bias }}=0-3 \mathrm{~V}$ & $\mathrm{U}_{\text {bias }}=3-10 \mathrm{~V}$ & $\mathrm{U}_{\text {bias }}=10-20 \mathrm{~V}$ \\
\cline { 2 - 6 } & $\mathrm{W}, \mu$ & $\mathrm{W}, \mu$ & $\mathrm{N}_{1}, 10^{20} \mathrm{~m}^{-3}$ & $\mathrm{~N}_{2}, 10^{21} \mathrm{~m}^{-3}$ & $\mathrm{~N}_{3}, 10^{21} \mathrm{~m}^{-3}$ \\
\hline 10 & $0.8-2.87$ & $2.87-5.03$ & 2.7 & 1.03 & 1.86 \\
\hline 30 & $0.8-3.23$ & $3.23-5.0$ & 2.14 & 1.13 & 2.5 \\
\hline 100 & $0.82-3.36$ & $3.36-5.17$ & 2.14 & 1.05 & 2.4 \\
\hline 300 & $0.87-3.3$ & $3.3-5.13$ & 2.18 & 1.03 & 2.04 \\
\hline 600 & $1.15-3.45$ & $3.45-5.24$ & 2.1 & 1.02 & 2.14 \\
\hline 1000 & $1.43-3.45$ & $3.45-5.0$ & 2.7 & 1.08 & 1.86 \\
\hline
\end{tabular}




\section{Conclusions}

It is shown that increase of small AC signal frequency $(10$ $\mathrm{kHz}-1 \mathrm{MHz}$ ) superimposed on DC bias voltage $\mathrm{U}_{\text {bias }}$ (positive potential is applied to $\mathrm{n}-\mathrm{Si}$ substrate) results in the decrease of measured barrier capacitance within interval 0 $3 \mathrm{~V}$ of micropixel avalanche photodiodes with deeply buried pixels. By further increase of voltage up to $20 \mathrm{~V}$ the magnitude of barrier capacitance does not depend on the frequency.

The width of space-charge layer $\mathrm{W}$ calculated from capacitance measurements varies from 0.8 up to 2.9 microns at $\mathrm{U}_{\text {bias }}=0-3 \mathrm{~V}$ and frequency $\mathrm{f}=10 \mathrm{kHz}$ and rises from 1.4 up to 3.45 microns at $\mathrm{f}=1 \mathrm{MHz}$. By the increase of voltage from 3 up to $20 \mathrm{~V}$, the width of space charge layer $\mathrm{W}$ reaches its maximum value of 5 microns and also doesn't depend on the AC signal frequency. The concentration of ionized acceptors in epitaxial layer has been calculated. It is shown that it varies weakly with AC signal frequency.

It is established, that for correct measurement of MAPD capacity with deeply buried pixels it is necessary to carry out measurements at low alternating signal with the frequency not more $100 \mathrm{kHz}$.

\section{REFERENCES}

[1] Z.Y. Sadygov. Microchannel avalanche photodiode, Russion Patent №2316848, priority from 01.06.2006.
[2] N.V. Anfimov, I. Chirikov-Zorin, A.A. Dovlatovet. al. Beam test of Shashlyk EM calorimeter prototypes readout by novel MAPD with super high linearity, Nucl. Instr. Meth. A. 617, 78-80, 2010.

[3] Z.Y. Sadygov, F. Zerrouk, A.A. Dovlatovet. al., Performance of new micro-pixel avalanche photodiode from Zecotek Photonics, Nucl. Instr. Meth. A, 610, 381-383, 2009.

[4] Z.Y. Sadygov et.al. Three advanced designs of micropixel avalanche photodiodes: Their present status, maximum possibilities and limitations, Nucl. Instr. Meth. A, 567, 70-73, 2006.

[5] Z.Y. Sadygov, A.G. Olshevski, A.A. Dovlatov et.al. Microchannel avalanche photodiode with rapid recovery time of parameters, Pisma v JTF, 39, №11, 7-12, 2013.

[6] Z.Y. Sadygov et.al. Technology of manufacturing micropixcel avalanche photodiodes and a compact matrix of their basis, Physics of Particles and Nuclear Letters 10, № 7, 780-782, 2013.

[7] Z.Y. Sadygov, A.G. Olshevski, A.A. Dovlatovet. al. Microchannel avalanche photodiode with wide range of linearity, Pisma v JTF, 36 v. 11, 83-89,2010.

[8] A.A. Dovlatov, Photoelectrical properties of micropixel avalanche photodiodes, PhD Thesis, 30.11.2011, 165

[9] Z. Y. Sadygov et.al. On features of potential distributional in avalanche photodiodes with deeply buried pixels, Fizika XIX, № 2, Sect. En, 17-19, 2013.

[10] E.A. Jafarova, Z. A. Iskenderzade, A.A. Dovlatov, L.A. Alieva, E.S. Tapdygov. Capacitance properties of micropixel avalanche photodiodes, Transactions of Azerbaijan National Academy of Sciences, Physics and Astronomy v.XXXII, № 2, 526-533, 2013. 